\title{
LA IMAGEN CARTOGRÁFICA DEL ESTRECHO DE MAGALLANES EN LAS DOS PRIMERAS DÉCADAS QUE SIGUIERON A SU DESCUBRIMIENTO
}

MATEO MARTINIC B. ${ }^{a}$

\section{RESUMEN}

El hallazgo y navegación del paso interoceánico a través del Nuevo Mundo por Fernando de Magallanes en 1520, una vez que fue conocido por las autoridades españolas tras el retorno de la nao Victoria en septiembre de 1522, fue valorado como un hecho geográfico de la mayor importancia para la corona castellana. En consecuencia, y sobre la base de la precaria información aportada por los sobrevivientes del histórico periplo mundial, pudo situárselo de inmediato en los mapas que brindaron una nueva y ampliada imagen del planeta según se lo venía conociendo. En este artículo se consideran las etapas y formas de una evolución cartográfica desde el sencillo bosquejo inicial hasta la más elaborada expresión particular del tempranamente célebre canal, obtenida al cabo de dos décadas de paciente recolección de noticias sobre el mismo, y de una cada vez más cuidadosa y fidedigna tarea profesional.

PALABRAS CLAVE: estrecho de Magallanes, cartografía, Patagonia, Tierra del Fuego.

\section{CARTOGRAPHIC IMAGE OF THE STRAIT OF MAGELLAN DURING THE FIRST TWO DECADES FOLLOWING ITS DISCOVERY}

\begin{abstract}
The discovery and navigation of the interoceanic passage through the New World by Fernando de Magallanes in 1520, once it was known by the Spanish authorities after the return of the nao Victoria in September 1522, was valued as a geographical fact of the greatest importance for the Castilian crown. Consequently, and on the basis of the precarious information provided by the survivors of the historical world tour, it was able to be placed immediately on the maps that provided a new and enlarged image of the planet as it was being known. This article considers the stages and forms of a cartographic evolution from the simple initial sketch to the most elaborated particular expression of the early famous channel, obtained after two decades of patient news gathering about it, and of an increasingly careful and reliable professional task.
\end{abstract}

KEY WORDS: Strait of Magellan, cartography, Patagonia, Tierra del Fuego.

Profesor Titular y Emérito, Universidad de Magallanes. Investigador Centro de Estudios del Hombre Austral, Instituto de la Patagonia, Universidad de Magallanes, Punta Arenas. \mateo.martinic@umag.cl 


\section{INTRODUCCIÓN}

Las semanas y meses que siguieron al arribo a Sevilla el 8 de septiembre de 1522 de la nao Victoria y sus dieciocho tripulantes -todo lo que restaba de la Armada de las Molucas que había zarpado de allí tres años antes al mando de Fernando de Magallanes en demanda de las islas homónimas por la ruta del occidenteal cabo de un larguísimo periplo que les había permitido circunnavegar el planeta, debieron ser sorprendentes y conmocionantes en la medida que se difundió la noticia del retorno y de lo logrado. Para la corona castellana, en especial, el viaje que había tenido tan crecido costo en vidas humanas, barcos y bastimentos, había sido gratificante por demás con su resultado: ¡Se había alcanzado hasta la rica tierra de la especiería por un camino distinto al de los portugueses $y$, por lo tanto, se podía plantear el reclamo sobre su jurisdicción!

Prepararse para fundar y defender ese anhelo, que se entendía era un derecho, pasó a ser, entonces, cosa de Estado, según debieron verlo y considerarlo el rey-emperador Carlos y sus consejeros, y otras altas autoridades del reino, materia de suyo importante al entenderse también que el vecino reino lusitano contestaría ese reclamo y procuraría afirmar el propio sobre un territorio al que había accedido con anterioridad. Los documentos escritos y dibujados fueron vistos como piezas de alto valor y determinantes, los últimos en particular -planos y mapas-, para la argumentación con que debería defenderse el reclamo para probar que la situación geográfica de aquellas codiciadas tierras, al parecer, las dejaba del lado español, esto es, al oriente del antimeridiano delimitatorio según lo pactado entre ambos reinos antes en Tordesillas.

Planos y mapas, genéricamente cartas en el lenguaje de la época, había que elaborarlos en forma complementándolos con las nuevas y estupendas informaciones geográficas aportadas por la empresa exploratoria de marras, cosa que exigía una especial actividad y dedicación a un capacitado conjunto de geógrafos y cartógrafos.

Tal debió ser, sin duda, el contexto motivador que debía inspirar la labor de esos técnicos bajo el apoyo y amparo de la Casa de Contratación de Sevilla, centro en el que hacía lustros se mantenía al día el Padrón Real o carta maestra de la geografía del mundo, a base de las informaciones paciente y cuidadosamente recogidas, colectadas $\mathrm{u}$ obtenidas de cualquier forma por gente al servicio de la monarquía castellana. De esa manera la memoria oral o gráfica de que podía disponerse al retorno de cada viaje al Nuevo Mundo cobraba un grandísimo valor y merecía, por tanto, la atención, la diligencia y el talento de los especialistas finales: los cartógrafos.

La gran travesía de 1519-1522 con su asombroso resultado había permitido comprender, sin razón alguna para dudar, la redondez y tamaño de la Tierra y calcular cada vez con mejor aproximación la proporción de tierras y mares en su inmensidad, y situarlos en los mapas para entender mejor tan trascendente novedad para la humanidad. En particular, las cartas a construirse debían reflejar conocimiento, situaciones y dimensiones de los sectores más determinantes en la nueva realidad, como eran el paso a través del Nuevo Mundo en la ruta por los grandes mares del Norte y del Sur, la magnitud y características de este último vastísimo espacio y la ubicación dentro del mismo de las cotizadas islas de las especias, de modo tan preciso como para asegurar su inclusión en la parte española delimitada por el antimeridiano de Tordesillas.

De estos tres sectores nos interesa el primero, en tanto que pieza clave en la futura ruta de exploración, explotación y dominio que debería plantearse como cosa subsiguiente en el nuevo cuadro de situación de las cosas del mundo. El estrecho encontrado por Fernando de Magallanes era entonces una posición estratégica excepcional como paso obligado en la futura ruta española a las Molucas. Debía ser descrito y situado en el inmenso espacio geográfico revelado según los datos disponibles, todo ello debidamente cartografiado para uso de navegantes, geógrafos, políticos, diplomáticos y empresarios económicos, compleja trama que, según sabemos, ya venía conformándose y desarrollándose desde cosa de un siglo antes cuando los portugueses, con el príncipe Enrique de Alvis como inspirador, habían iniciado la época de las grandes exploraciones marítimas y de la expansión geográfica del mundo.

Hasta entonces, por lo demás, los mapas del mundo reflejaban el grado de conocimiento que se poseía y que, en el caso del Nuevo Mundo, era 
de total ignorancia en lo que se refería a su zona meridional ${ }^{1}$.

Situado en este contexto histórico el punto, pasamos a considerarlo debidamente.

\section{DE LA OBSERVACIÓN A LA REPRESENTACIÓN DE LO OBSERVADO}

¿Memoria oral o documentos? Lo que se supo y conservó después del retorno de los sobrevivientes del gran viaje.

Así pues, recoger, revisar y valorar la información que aquellos supérstites de la gran empresa magallánica pudieron brindar debió ser cosa inmediata, sino urgente, por necesaria. Pero en verdad, ¿cuánto de información novedosa y provechosa trajeron esos afortunados retornados (más lo que agregarían los pocos compañeros que habían quedado rezagados y desperdigados por distintas causas durante la etapa final de la travesía)? Poco, quizá demasiado poco de momento, según pasamos a considerarlo.

Importa de partida conocer el escaso acervo informativo disponible en la coyuntura, saber de las causas de tal carácter y ver cómo la necesidad de que se trata pudo satisfacerse porque, ya se sabe, el tiempo apremiaba.

Tocante a la información documental de primera mano hasta ahora conocida sobre lo acontecido en el histórico periplo mundial iniciado y bien adelantado por Magallanes, concluido por Elcano, nos hemos preocupado del punto en nuestra obra más reciente de modo extenso ${ }^{2}$, por lo que cabe únicamente resumirlo. Apenas ocho piezas escritas por algunos de los participantes en el viaje, cuya extensión referencial al estrecho de Magallanes va desde seis páginas (Pigafetta) a solo una línea (Pancaldo), acervo de testimonios prima facie complementado por otros cuatro relatos de época recogidos por cronistas que trataron a algunos de los tripulantes sobrevivientes (Brito, Transylvanus, Angheria y Herrera).

1 Buena prueba de lo que se afirma se tiene en el mapamundi Orbis typus universalis ivxta hydrographorum traditionen exactissime depicta de 1522, cuyo autor y editor es el cartógrafo de Estrasburgo Lorenz Fries (Lautentius Frisius o Laurentio Frisio), en que se muestra América con su parte austral interrumpida a partir de los $40^{\circ}$ de latitud sur.
Toda la documentación que pudo hallarse al tiempo de la captura de la nao Trinidad en Tidore por parte de los portugueses fue secuestrada, y si algo escapó a la requisa entonces también lo fue más tarde (caso de la Victoria), de manera que muy poco de lo escrito (o dibujado) durante la travesía pudo retornar a Europa con los sobrevivientes de la misma. De todo ello, importa consignarlo, en el presente conocemos todavía menos porque varios de esos testimonios se perdieron por distinta causa hace ya mucho tiempo.

Ahora bien, la documentación escrita de la que pudo disponerse al tiempo del regreso de la Victoria (octubre de 1522) y que daba cuenta de lo sucedido y visto, más las relaciones recogidas de los tripulantes, no bastaba para elaborar mapas descriptivos de las nuevas regiones geográficas descubiertas. En lo que concierne al Estrecho, las impresiones de los diferentes recordantes concordaban en su estimación de longitud, un centenar de leguas poco más o menos (medida española de la época) y en la distinta amplitud observada entre sus orillas norte y sur, ora muy angosto, ora más ancho alternativamente, con cantidad de islas grandes y pequeñas en su curso, con riberas de tierras bajas y amarillentas y de aparente sequedad por el lado del oriente, y costas abruptas con altas montañas cargadas de nieve, en parte revestidas de bosques frondosos y olorosos, parajes abundantes en ríos y arroyos, leña y hierbas comestibles; con abundancia de pescado y mariscos por el occidente, ámbito ventoso y revuelto en sus tiempos atmosféricos y con mucho, muchísimo frío, en fin. Todo esto estaba, bien como memoria individual y colectiva sobre esa parte del gran periplo mundial, bastante para ilustrar a una autoridad o a un auditorio, pero que de poco servía para elaborar un plano que mostrara sus características más notorias, según se las había observado, como para instruir a los que vinieran después por la misma ruta, información valiosa que era lo que se necesitaba. Para ese efecto solo eran

Este poco conocido mapa se conserva en la Sächsischen Landesbibliothek de Dresde, Alemania.

2 Cfr. Martinic, 2016. Una travesía memorable. Hallazgo y navegación del estrecho de Magallanes. Aguas Magallanes, Punta Arenas. Apéndice, pp. 219 y siguientes. 
útiles los mapas, y cuanto más exactos, esto es, ajustados a la realidad geográfica, mejor.

Así, entonces, los bosquejos o esquicios realizados durante el trayecto cobraron un valor informativo insustituible por cuanto únicamente de ellos, debe aceptarse, podía obtenerse una información inteligible fiable en los detalles pertinentes para construir una carta que proporcionara una visión siquiera preliminar sobre lo observado y registrado como para que después otros pudieran repetir en sucesivas navegaciones aquella histórica experiencia.

Aunque en todo lo escrito e investigado sobre la misma nunca, que sepamos, se han mencionado papeles de la especie, no debiera caber duda de que algún o varios planos pudieron ocultarse a los portugueses y llegar finalmente a manos de los agentes u oficiales de la Casa de Contratación, en Sevilla. Lo prueban así el mapa de Turín, al que más adelante haremos cumplida referencia, y la gran carta del mundo (planisferio) utilizada por los españoles en las conferencias de Badajoz-Elvas en 1524 , ocasión en que castellanos y portugueses expusieron y discutieron larga e infructuosamente acerca de la ubicación del antimeridiano de Tordesillas $y$, por consecuencia, para verificar la ubicación precisa de las islas Molucas. Una y otra valiosas piezas fueron elaboradas por maestros calificados de la cartografía, teniendo como fuente documental de primera mano y por ello fiable, es nuestra hipótesis, de los planos o dibujos levantados durante el viaje (en el caso, durante la travesía del estrecho de Magallanes) y cuidadosamente conservados para el mejor servicio real. Otra cosa es que, por razones que se desconocen, tales preciosos documentos acabaran extraviados y nada de ellos ha llegado hasta nosotros.

Abundando en el punto, ocurre que en todo lo investigado y lo mucho escrito sobre la memorable expedición magallánica, aunque se ha mencionado repetidamente la existencia de papeles, se ha dado a entender, o se ha supuesto, que los mismos fueron solo documentos escritos, en forma de anotaciones de bitácora, diarios de vida o registros varios, y no así de planos con

3 Zweig, 1972. Magallanes. Historia del primer viaje alrededor del mundo. Editorial Juventud S.A., Barcelona. Véase el Informe sobre el coste de la flota en página 263 y siguientes en el que, entre otras muchas partidas e ítemes, bosquejos de levantamientos y trazados de cartas con sus accidentes más notables, suficientes como para permitir tener de visu una idea sobre lo encontrado. De alli que cabe preguntarse acerca de si tales documentos pudieron de verdad existir. Sí que lo fueron y pruebas al canto.

Primero, tenemos la constancia de haberse embarcado en las naos al tiempo de la partida desde Sevilla elementos para tal faena gráfica, como pergamino, y además cuero para prepararlo por si hiciera falta. Segundo, añadimos los varios planos cuya autoría se ha atribuido a Pigafetta y que más allá de su calidad representativa sugieren haber sido elaborados teniendo a la vista originales realizados por terceros. Y tercero, por si faltara, contamos con la propia mención del cronista lombardo, quien así lo da a entender claramente en su afamada relación.

Para afirmar la primera probanza basta la referencia explícita contenida en el libro Magallanes. Historia del primer viaje alrededor del mundo del ilustre escritor Stefan Zweig, sin duda la más conocida de las obras referidas al gran navegante y su afamada empresa, exceptuada la relación de Pigafetta ${ }^{3}$. Respecto de la segunda, cabe remitirse a las ilustraciones incluidas en las distintas ediciones de la relación de Antonio Pigafetta a contar de 1525. Y en cuanto a la tercera, la referencia explícita se contiene en la parte del famoso escrito en que se narra el supuesto extravío de la nao San Antonio en el Estrecho (en verdad una deserción) y de las diligencias ordenadas por Magallanes para su búsqueda:

envió atrás el capitán general a la Victoria, hasta la misma entrada del Estrecho, porque viese si andaba por allí; y que de no encontrarla, clavase una bandera sobre algún montículo, con una carta metida en ella y ahincada en tierra junto al mástil; de forma que con descubrirla, encontrando la carta, supiesen el rumbo que seguíamos. Porque esas eran nuestras órdenes estipuladas para el caso de que una nave se distanciase de las otras. Dos

se da cuenta del gasto de 1.764 maravedíes pagados por la adquisición de dos docenas de piezas de pergaminos ( $p$. 270). 
banderas con cartas se clavaron esta vez. Una, sobre un alcor [colina] de la primera bahía i la otra, en un islote de la tercera materialmente lleno de lobos marinos y grandes pájaros ${ }^{4}{ }^{5}$.

Cabe explicar el término "carta" empleado por Pigafetta, debiera entenderse en la forma que era corriente en la época, esto es, como sinónimo de mapa o plano ${ }^{6}$ y no como un mensaje escrito con instrucciones sobre cómo navegar aguas adentro del Estrecho hasta ubicar a las otras naos de la armada. Un plano con un boceto o esquicio que figurara las costas y los accidentes más notorios en ellas avistados nos parece que debía ser cosa de mayor utilidad para el objeto de que se trataba, que una misiva con instrucciones acerca de cómo y por dónde navegar. La primera modalidad nos parece mucho más práctica y útil para un hombre de mar acostumbrado a su uso (caso del capitán o piloto a quien iba destinada).

Debe aceptarse, además, que el levantamiento de planos durante esa travesía (como en tantas otras de aquellos tiempos) era cosa obligada, como se infiere del conocimiento del material y elementos enmarcados para tal efecto, según se ha visto. Nos confirma en la interpretación que postulamos, asimismo, el mapa del Estrecho elaborado por Pigafetta (o atribuido a él), que más allá de su paupérrima fidelidad geográfica, es evidente que únicamente pudo ejecutarse o copiarse con más precisión teniendo a la vista uno o varios planos realizados por gente con mayor conocimiento del oficio. Tal, conjeturamos, pudo hacerse en el transcurso o hacia el fin de esa etapa determinante del gran viaje.

Para concluir nuestra argumentación señalamos que las referencias geográficas compulsadas en los escritos que han llegado

4 Pigafetta, 1985. Primer viaje alrededor del mundo. Edición de Leoncio Cabrero, Historia 16, Madrid, pp. 73 y 74. Lo destacado es nuestro.

5 Las referencias geográficas las entendemos hechas al monte Dinero (costa norte del Estrecho), en el primer caso, y a uno de los dos islotes situados en el principio del sector central del gran canal (procediendo de oriente a poniente), hoy nombrados Magdalena y Marta.

6 Acepción que aún mantiene vigente el Diccionario de la Real Academia Española de la Lengua.

7 Motivado por los resultados del viaje de Magallanes, el hasta el presente -en los casos que las hayson vagas y/o mínimas $y$, en cualquier caso, insuficientes como para permitir una idea acerca de la conformación del paso interoceánico y sus características y rumbos, por lo que puede sostenerse que el primer cartógrafo que elaboró un mapa del Estrecho debió necesariamente tener a la vista planos o esquicios dibujados durante la travesía fretana, pues solo así pudo obtenerse la imagen primeramente conocida.

\section{CARTOGRAFÍA FRETANA INICIAL (1522-1524)}

Precisando en la materia de la cartografía que nos ocupa, solo tenemos certidumbre sobre cuatro mapas que dan cuenta del hallazgo geográfico realizado por Fernando de Magallanes en la parte austral de América. Uno, debido a Pedro Reinel; un segundo es el ya mencionado mapa de Antonio Pigafetta; el tercero, una carta planisferia de autor desconocido; $y$, por último, el planisferio conocido como Padrón Real de Turín, atribuido a Nuño García de Toreno ${ }^{7}$.

Respecto del mapa de Reinel, o de lo que del mismo se conoce actualmente en realidad, se trata de la mitad de un planisferio que representa el hemisferio austral del mundo, pieza que se encuentra depositada en el archivo del museo y palacio Top Kapi Sarayi de Estambul, y que fue dada a conocer por el geógrafo francés Marcel Destombes en el transcurso del Congreso Internacional de Geografía realizado en Âmsterdam en 1938. Los estudios realizados a su vista han permitido concluir que la pieza encontrada corresponde a parte de un planisferio elaborado en 1519 por el maestro portugués Pedro Reinel, a la sazón trabajando para la Casa de Contratación, con la colaboración de su hijo Jorge. Este planisferio según la opinión del

cosmógrafo Juan Vespucio, de la Casa de Contratación, elaboró hacia 1523 su mapa Totivs Orbis Descriptio, trazado en proyección azimutal equidistante. En esta pieza el cartógrafo incorporó los descubrimientos geográficos del navegante portugués, pero en el hemisferio austral (que se muestra dividido en dos mitades) la representación del meridión americano aparece incompleta y desactualizada, lo que hace a esta pieza irrelevante para nuestro estudio. Véase la ilustración de página 94 en la obra de Martín Merás, 1993. Cartografía Marítima Hispana. La imagen de América. Lunwerg Editores, Barcelona. 
historiador naval Ricardo Cerezo Martínez, que compartimos, habría integrado el conjunto de cartas de navegación preparadas especialmente para la expedición de Fernando de Magallanes, y que éste habría distribuido entre las diversas naos de su armada para el uso de los respectivos capitanes y pilotos. Así, entonces, una de estas piezas llegó a la nao San Antonio, después desertora de la armada en el Estrecho, y con ella retornó a España en 1521. Sus tripulantes pudieron así aportar las primeras informaciones sobre lo acontecido en la expedición, incluyendo lo explorado hasta el tiempo de su huida.

Pues bien, sobre esta base, alguien, quizá por encargo del cartógrafo autor original, habría agregado en la parte del mapa referida a lo conocido hasta entonces del continente americano un trazado que extiende la tierra firme hacia el suroeste hasta los $53^{\circ} \mathrm{S}$, y que termina en una suerte de golfo con una isla en su interior, a cuyo largo corre la leyenda Hesta terra descobrio Fernando de Magalhaes. El carácter de añadido al trazado original es evidente, pues denota una mano ejecutora distinta a la del cartógrafo dibujante anterior y con un colorido pardo-verdoso que difiere de la tonalidad del resto de la pieza. La mención toponímica consignada corresponde a algo que únicamente pudo ser conocido en España al arribo de la San Antonio ${ }^{8}$. El alejamiento de este buque impidió a sus tripulantes enterarse del feliz término de la exploración al Estrecho y, por ello, desconocer sus características geográficas reales, circunstancia reflejada en el dibujo añadido a la pieza del museo de Top Kapi Sarayi, que representa el sector por ellos conocido. Así pues, este mapa, sin embargo de su interés, queda fuera de la materia de este trabajo al no incluir la representación completa del estrecho de Magallanes.

El plano de Pigafetta también se excluye, no obstante cumplir el requisito mencionado, por el hecho de tratarse, así lo entendemos, de una representación meramente aproximada cuyo objeto fue simplemente el de complementar la relación de lo acontecido durante el memorable

8 Martinic, 2005. El protomapa de Chile. Boletín de la Academia Chilena de la Historia, 114, 175-182.

9 Barreiro-Meiro, 1975. El Pacífico y el estrecho de Magallanes en la cartografía del siglo XVI. En A viagem viaje. Nos mueve a esa interpretación la manifiesta desproporción que se observa entre los sectores geográficos representados (en conjunto la mitad austral del continente sudamericano) y la ausencia de una mejor correspondencia entre los accidentes litorales más notorios observados durante el viaje y la realidad. Un hombre de mar no habría podido ser tan chapucero en el dibujo, por más que su competencia fuera ajena a la de un cartógrafo, por lo que debiera aceptarse que el ejecutor del mapa, fuera o no Pigafetta, únicamente quiso ilustrar con tan burdoy desprolijo trazado a cuantos habrían de leer la relación de lo acontecido (Fig. 1). A idéntica conclusión ha llegado otro historiador, Roberto BarreiroMeiro, al afirmar que los mapas de Pigafetta.... más que cartas, son dibujos abocetados de las partes más interesantes por las que pasaron ${ }^{9}$. Ello no significa, debe aceptarse igualmente, una descalificación para la importancia que de suyo tiene el mapa en cuestión para la historia cartográfica, sino únicamente su exclusión como pieza de pertinencia en la materia que nos interesa, o sea, en la mejor imagen inicial del estrecho de Magallanes.

El tercer mapa mencionado para el asunto que nos ocupa es un documento extraviado hace siglos y respecto del que solo podemos brindar una buena referencia. Se trata de un planisferio que fue construido especialmente para ser presentado por los negociadores castellanos a sus pares lusitanos durante la reunión de Badajoz-Elvas en mayo de 1524. Se le recuerda con una doble denominación, la de Carta de navegación de Castilla hasta los Malucos, o bien como Padrón nuevamente hallado por los que vinieron de la Especiería. De autor desconocido, hay consenso entre los historiadores en cuanto a que el mapa fue elaborado durante el año precedente a la mencionada reunión sobre la base de los antecedentes reunidos por la Casa de Contratación. De esta pieza se sabe que contenía el cabo de San Agustín en el Brasil, en 8 grados de latitud S. y en 20 grados de longitud hacia el O. de la isla de San Antonio; y que estaba asentada toda la costa hasta el estrecho de los

de Fernao de Magalhaes e a questao das Molucas. Actas do II Coloquio Luso-Espanhol de Historia Ultramarina. Lisboa, p. 523. 


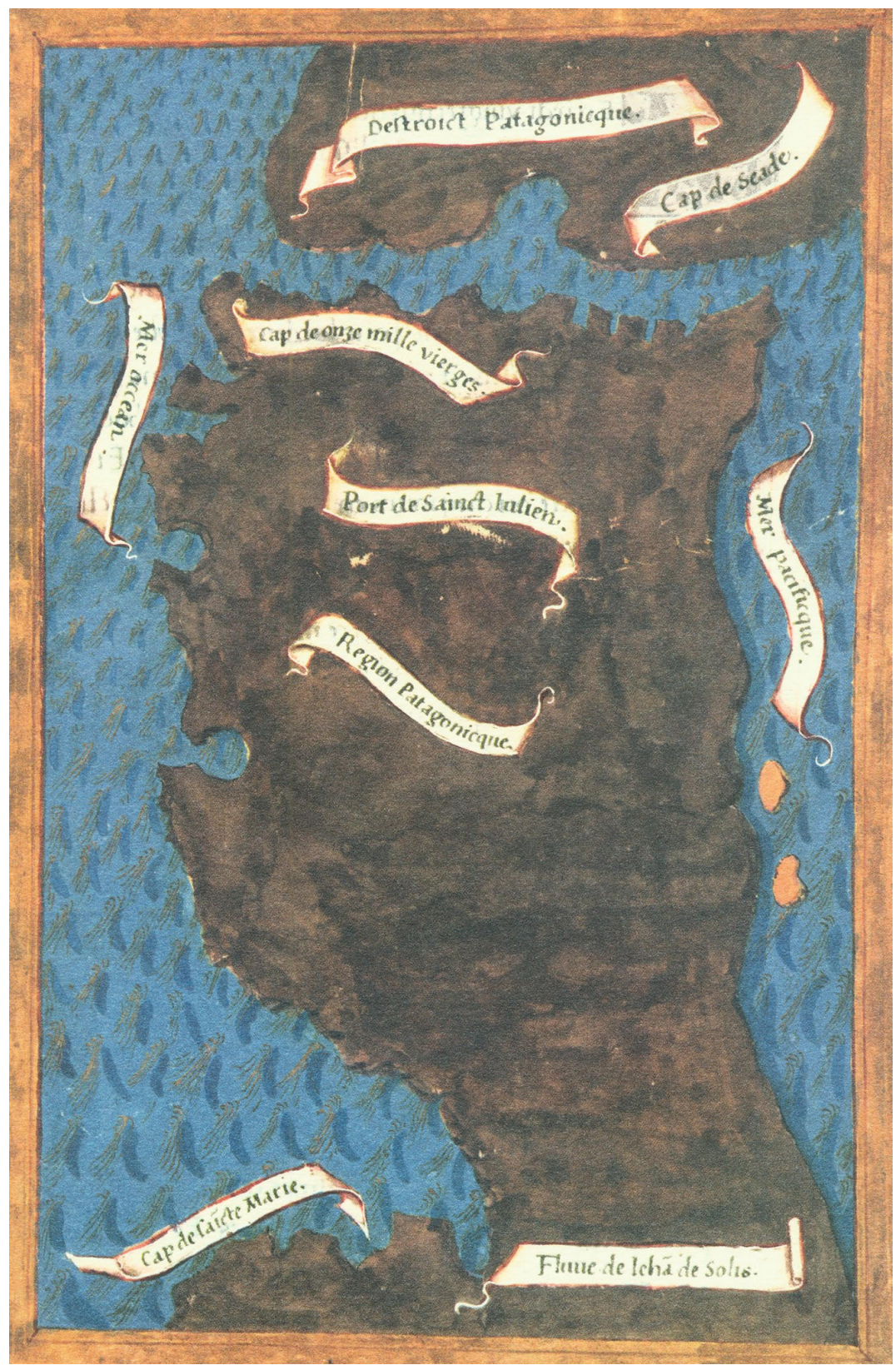

Fig. 1. Antonio Pigafetta, Journal of Magellan's Voyage, ca.1525.

Cortesía de The Beinecke Rare Book \& Manuscript Library, Yale University, New Haven.

Malucos [de Magallanes] cuya boca es en 52 1/2 grados latitud S. y 4 1/2 grados más al $\mathrm{O}^{10}$.

Resta del listado precedentemente mencionado solamente el planisferio conocido

10 Fernández de Navarrete. Colección de viajes, y Descubrimientos que hicieron por mar los españoles desde fines del siglo XV. Tomo IV, doc. XXXVIII, p. 330, como Padrón Real de Turín, única pieza de la cartografía fretana temprana que ha llegado hasta nosotros. Esta circunstancia y su calidad específica le confieren al mismo un carácter excepcional por

citado por Cerezo Martínez, 1994. La cartografía náutica española en los siglos XIV, XV y XVI. Consejo Superior de Investigaciones Científicas, Madrid, p. 180. 
su fiabilidad representativa, en grado inicial, del estrecho descubierto por Fernando de Magallanes en la memorable travesía exploratoria que tuvo comienzo el 21 de octubre y feliz término el 28 de noviembre de 1520. Su indudable mérito justifica una consideración particular más extensa.

\section{EL PLANISFERIO DE TURÍN}

\section{Descripción del mapa y \\ consideraciones sobre su autoría}

Esta notable pieza, la única de su especie construida durante el bienio que siguió el retorno de la Victoria que se ha conservado hasta el presente, tiene el mérito particular de ser una copia del padrón real -la carta maestra confeccionada sobre la base de los datos aportados por las sucesivas exploraciones hacia ultramar- elaborado por la Casa de Contratación, con uso de la información geográfica y náutica obtenida en el viaje de Magallanes. Es propiamente una carta universal o mapamundi de gran tamaño $(274 \times 124$ $\mathrm{cm})$ compuesta por la unión de tres trozos de pergamino, elaborada a mano e iluminada con tonalidad amarillo-verdosa apreciable no obstante la decoloración y el avejentamiento del cuero ocasionados por el mero transcurso del tiempo. Está decorada en el interior de América y África con la representación de selvas y montañas en colores verde $y$ pardo, respectivamente, y con una profusión de rosas de los vientos distribuidas por todos los océanos desde las que arrancan líneas loxodrómicas, amén de una mayor en el sector del Pacífico de la línea ecuatorial. Incluye, asimismo, la graduación de la latitud y la longitud en idéntica medida. Todo el conjunto muestra una gran sobriedad, llamativa para una época en que la ornamentación en piezas del género podía alcanzar ribetes exagerados. Adquirida por compra o donación, perteneció a la casa ducal, después real, de Saboya, y se guarda en la Biblioteca Real de la ciudad de Turín.

Representa todo el orbe conocido en la época: Eurasia, que ocupa el ángulo superior derecho del mapa; África al sur de aquella y el Nuevo Mundo, en el centro, figurando incompleto con una gran parte de Sudamérica (sectores fronteros al Caribe y Atlántico) y su continuación continental hacia el septentrión, pero restringida a las zonas todavía en exploración y poco definidas, $y$, por fin, las islas del Caribe. En la parte izquierda del pergamino se muestran las tierras insulares del oriente lejano recién descubiertas, entre ellas las Filipinas, además de las Molucas, separadas del Nuevo Mundo por un vastísimo espacio conformado por el gran mar del Sur o Pacífico según la nueva denominación; el estrecho de Magallanes se sitúa en la parte inferior de América al centro de la carta planisferia. No aparecen la demarcación longitudinal acordada entre Castilla y Portugal en el tratado de Tordesillas ni el antimeridiano en la zona de las islas Molucas. Las dimensiones y ubicación de tierras y mares reflejan en lo ancho del mapa la certidumbre del nuevo tamaño reconocido para el planeta al cabo del memorable periplo magallánico.

El cuidadoso trazado de las líneas de la costa y la abundante toponimia que las jalona tierra adentro, en su caso como a las insulares, dan cuenta de su ejecución por una mano maestra, con prolijidad, arte y conocimientos profundos del oficio. El punto ha sido bien estudiado por especialistas expertos en la materia, principalmente el historiador italiano Alberto Magnaghi, quien concluyó en que la gran carta parecía ser más el fruto de una tarea colectiva, aunque sugirió una participación en grado preponderante de Juan Vespucio. Esta afirmación ha sido discutida por otros historiadores españoles contemporáneos, como Ricardo Martínez Cerezo y Luisa Martín Merás, por el primero en particular, los que sí convienen en la posibilidad de una labor en conjunto de cosmógrafos, sostienen que hay antecedentes $e$ indicios que permiten atribuir la paternidad de la obra al eximio maestro cartógrafo Nuño García de Toreno. Éste, en verdad, tenía suficiente competencia y prestigio como para dirigir tal faena especializada, no en vano era el piloto mayor de la Casa de Contratación desde 1519, cargo que desempeñaría hasta su muerte en 1526. Su experiencia, avalada por una cantidad de obras importantes del género, entre ellas la elaboración de 25 cartas expresamente preparadas para el viaje de Fernando de Magallanes, así como su prolijidad en el dibujo cartográfico, su habilidad como iluminador de pergaminos y su fama como innovador en la fábrica de cartas, justificaban su fama y avalan con suficiencia la interpretación hecha acerca de su posible paternidad de la pieza de que se trata. 


\section{El sector del Estrecho en el planisferio}

Sin embargo de la escala mayor que tiene el planisferio de Turín que de suyo exige una reducción en el detalle del trazado litoral de tierras, con omisión de características que pudieran hacerlo más comprensivo, en el caso de la representación del estrecho de Magallanes -junto con la gran dimensión reconocida al océano Pacífico conforman las grandes novedades del mapamundi-, de primera se aprecia el cuidado en la información que da cuenta de sus rumbos y rasgos geográficos más sobresalientes según se observara y anotara durante la travesía exploratoria.

En efecto, la forma de su desarrollo general presentada con una dirección en línea flectada a modo de una "V" abierta, da cuenta de la apreciación de esa circunstancia, noción afirmada además con las conocidas mediciones hechas por el piloto Francisco Albo, de la nao Trinidad, para fijar la situación del cabo Once Mil Vírgenes en la entrada oriental y para el cabo Deseado en la boca occidental, estimadas respectivamente en $52^{\circ} \mathrm{S}$ (52 $25^{\circ}$ S y $42^{\circ} 44^{\prime}$ S en la realidad), como el ángulo de inflexión en el sector central del paso, 53 dos tercios ( $54^{\circ} \mathrm{S}$ en la realidad). Además, son notorios los rasgos que definen los principales accidentes caracterizadores del canal, tal como lo recordaría la memoria de los tripulantes sobrevivientes, como son el golfo o saco marino del acceso oriental, las angosturas siguientes y su ensanche intermedio, el paso ancho del Estrecho y las islas de su cabezo, su división en dos vías dejando una tierra intermedia y la longitud rectilínea de la mitad occidental al cabo de la inflexión central, todo lo cual satisface ampliamente como representación inicial de una hidrografía compleja como es en su realidad la configuración fretana. Esta comprobación inicial no será superada en las siguientes representaciones cartográficas, excepción hecha de la brindada lustros más tarde por el mapa de Alonso de Santa Cruz, según se verá más adelante, sino hasta el tiempo del cambio de siglos, del XVI al XVII, con la elaboración de los mapas que recogieron las observaciones, registros y trabajos de los navegantes y cartógrafos holandeses. Para concluir, lo único que llama nuestra atención es la escasa toponimia

11 Esta primacía se reconoce heráldicamente en la divisa Prima in Terra Chilensis que integra el escudo de la región acompañante en una gran carta en que la misma superabunda, aspecto que se trata en particular más adelante. A propósito, el hecho de incluirse en este mapa algunos topónimos cuya paternidad debiera atribuirse a Fernando de Magallanes, v.gr. Tierra de Diziembre, nunca después repetidos en carta alguna, nos hace sospechar que entre las fuentes que informaron su elaboración debió haber, con certidumbre, algún plano hecho por el propio navegante o por alguien bajo su supervisión, pieza que debió extraviarse hace ya mucho tiempo.

La descripción precedente basta para calificar esta notable obra que hemos reconocido y que reiteramos como el documento fundacional de la cartografía nacional chilena, pues brinda con la mejor claridad posible para el tiempo de su ejecución, la primera información de un sector geográfico que más tarde integraría la totalidad del territorio de Chile ${ }^{11}$ (Figs. 2 y 3).

\section{LA SECUENCIA CARTOGRÁFICA 1525-1540. ALTOS Y BAJOS}

Las negociaciones luso-castellanas de Badajoz-Elvas en abril y mayo de 1524, bien se sabe, concluyeron en un fracaso al no ceder las partes en sus posiciones respecto de la ubicación geográfica de las codiciadas islas Molucas en relación con la línea del antimeridiano de Tordesillas, motivo preciso de la conferencia. Preocupado por ello el monarca español Carlos de Habsburgo, dispuso la realización de una nueva expedición a la tierra de las especias para afirmar la ventaja obtenida con la nueva ruta de acceso a la misma brindado por la empresa magallánica. Contando nuevamente con el apoyo financiero de la casa alemana Fugger, su jefatura se encomendó al comendador Francisco García Jofré de Loayza. Integrada la armada por siete naos, entre sus tripulantes se contaban cuatro de los antiguos compañeros de Magallanes: el principal de ellos era Juan Sebastián Elcano, en calidad de segundo comandante de la flota. La expedición zarpó del puerto de La Coruña el 24 de julio de 1525; su objetivo preciso era el de consolidar la jurisdicción reclamada sobre las islas de las especias e iniciar la explotación de sus valiosos recursos por cuenta hispana.

de Magallanes. 


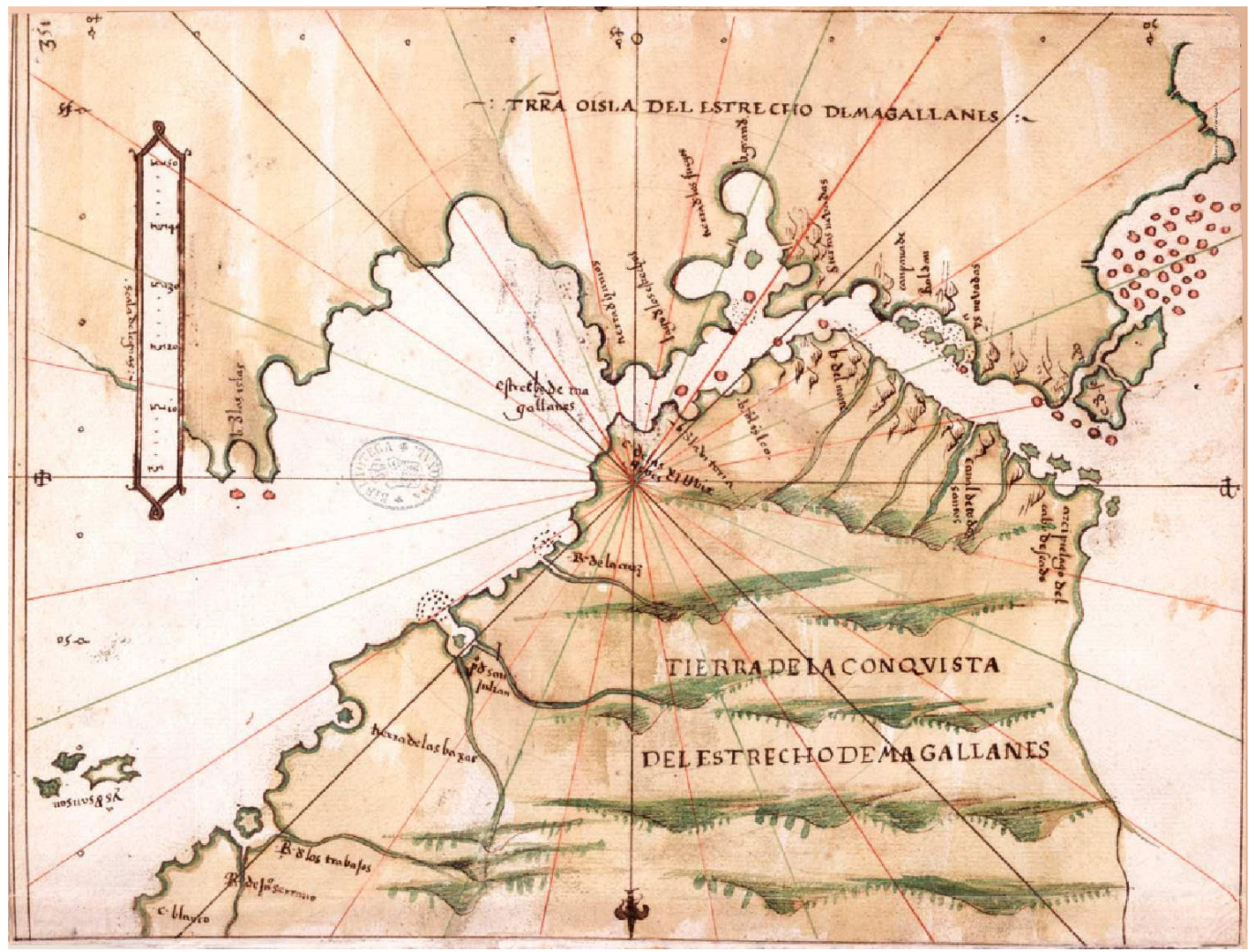

Fig. 2. Carta anónima de Turín, atribuida a Nuño García de Toreno, 1523. Biblioteca Real de Turín.

Mientras tal se obraba, en simultaneidad se dio comienzo a una sutil labor de propaganda diplomática destinada a impresionar a los soberanos de otros reinos europeos, en especial al principal poder de la época, el papado católico de Roma, acerca de la nueva realidad geográfica del orbe y de sus proporciones, como también para ilustrarlos sobre la presencia jurisdiccional de España en los nuevos territorios revelados por las exploraciones ultramarinas. Ello únicamente podía conseguirse con la elaboración cartográfica por su capacidad para dar cuenta de visu de la nueva situación. En esa información el paso interoceánico revelado en el sur de América -el Estrecho por antonomasia- era solo un dato, aunque importante, como referencia de ubicación.

Esa movida era ciertamente una cosa necesaria como complemento gráfico para una novedad sensacional como había sido la brindada tras la llegada de la Victoria luego de completar la circunnavegación del mundo, cuya noticia no demoró en difundirse para la información del ámbito ilustrado de Europa. Tal propósito se constataba con la circulación que había tenido y tenía la relación hecha por Maximiliano Transylvanus, secretario del rey-emperador Carlos, poco después que éste recibiera en Valladolid a Elcano y se informara por él y luego por el cronista Antonio Pigafetta sobre lo acontecido con la expedición magallánica. Tan impresionado como pudo estarlo el propio monarca, Transylvanus no demoró en participar a su padre, entonces arzobispo de Salzburgo, la estupenda novedad, provocando en su destinatario como en aquellos que por él mismo se impusieron una idéntica gran sensación, circunstancia que explica las sucesivas publicaciones de la carta en forma impresa en Colonia, París y Roma entre 1523 y 1525 . Un reciente hallazgo cartográfico sugiere que tal misiva pudo ser acompañada de un plano o boceto para su mejor entendimiento. Se trata del mapa elaborado hacia 1525 por el ya mencionado Lorenz Fries, médico y cartógrafo de la ciudad de Estrasburgo y titulado Tabula Moderna Alterius Hemisphaerii, en que se presentan las mayores novedades del 
periplo mundial de Magallanes, en el estrecho en la parte austral de América y el océano Pacífico ${ }^{12}$.

La iniciativa oficial de que se trata comenzó a manifestarse con la elaboración de dos cartas universales durante 1525. Una, la Carta del Navegare Universalissima et Diligentissima cuya autoría se ha atribuido al cosmógrafo portugués Diego Ribero, que integraba el elenco de profesionales de la materia en la Casa de Contratación, pieza valiosa regalada por Carlos $\mathrm{V}$ al cardenal Castiglioni, enviado papal a España con motivo del matrimonio del monarca con la princesa Isabel de Portugal. La otra, de la que no se conoce el título, fue ejecutada por Nuño García de Toreno por encargo regio y con idéntico propósito, ahora para obsequio al cardenal Juan Salviati, también invitado al acontecimiento. En ambos casos debiera aceptarse que el destinatario del sutil mensaje diplomático era el Sumo Pontífice, en Roma ${ }^{13}$. Una tercera carta universal que puede adscribirse al mismo interés propagandístico fue la preparada en 1526 por Juan Vespucio, otro de los calificados miembros del elenco de cosmógrafos de la corona hispana. Estas tres bellas piezas de la cartografía universal han llegado hasta nuestros días $^{14}$. De su contenido interesa la representación del estrecho de Magallanes, en cuanto materia de nuestro artículo.

Pese a la escala de los mapas, insuficiente para la observación de detalles, es posible apreciar que solo en el mapa de Ribero la figuración del Estrecho se asemeja al modelo original aportado por el planisferio de Turín, sugiriendo por ello la misma inspiración en el padrón real. En cambio, tanto en las cartas de García de Toreno (Castiglioni)

12 Cfr. Muller, 2012. Tabula Moderna Alterius Hemisphaerii. The oldest surviving map of the Pacific? The Globe. Journal of the Australian and New Zeland Map Society Inc., 71. Véase también Martinic, 2017. Tierra de Vespucio. Consideraciones sobre una curiosa representación del estrecho de Magallanes en la Tabula Moderna Alterius Hemisphaerii, un mapa recién conocido del siglo XVI. Magallania, 45(1), 5-14.

13 Esta conjetura la afirmamos con la decisión papal de ordenar la ejecución de una serie de grandes frescos murales referidos a las distintas partes del mundo entonces conocidas pintados en los muros de la gran galería de acceso a la Capilla Sixtina, por lo mismo conocida como Galería de los Mapas. Uno de estos frescos, que observamos en una visita hecha en 1976, representa a Sudamérica con el estrecho de Magallanes. Cfr. y de Vespucio (Salviati), muestran una imagen deformada del paso interoceánico tanto en rumbos como en accidentes geográficos, desajuste mucho más notorio en la grosera interpretación hecha por Fries $^{15}$

La secuencia cartográfica registrada permite mencionar otros tres mapas universales cuya fecha de elaboración se ha situado en o hacia 1527: el Mapamundi en seys pliegos y al fin occidente del mundo, de autor desconocido y que perteneció a la Biblioteca Colombina, de la que desapareció hace ya largo tiempo. Esta sería la primera pieza impresa de la que hay conocimiento con los nuevos descubrimientos geográficos. Luego está el mapamundi confeccionado por el maestro genovés Vesconte de Maggiolo que, en lo que interesa, muestra al Estrecho con un trazado simplificado en sus detalles con relación al patrón de 1523. La tercera carta es poco más que un bosquejo planisférico toscamente elaborado por Robert Thorne, comerciante inglés radicado en Sevilla, preparado para informar a su soberano Enrique VII acerca de los recientes descubrimientos españoles, que incluye una paupérrima y deformada imagen del estrecho de Magallanes.

Se prosigue con otras tres cartas de idéntica autoría, del maestro Diego Ribero, y presentadas con un título semejante Carta Universal en que se contiene todo lo que del mundo se ha descubierto hasta agora, la primera fechada en 1527 y las otras dos en 1529, que se conservan en la Thuringische Landesbibliothek de Weimar, la primera y la última, y en la Biblioteca Apostólica Vaticana, la segunda. Además de compartir la misma magistral autoría en la visión general del orbe conocido, en

Barber (Ed.), 2005. The Map Book, Levenger Press, New York, pp. 122 y 123

14 Estas piezas se conservan, respectivamente, en el Archivo de los marqueses de Castiglioni Mantua; en la Biblioteca Laurenziana, Florencia, y en The Hispanic Society of New York.

15 De esta secuencia representativa se ha excluido un mapa de la costa patagónica y del estrecho de Magallanes atribuido al piloto Juan Sebastián Elcano, que habría sido realizado hacia 1523, en atención al carácter apócrifo de la obra, opinión compartida por la mayoría de los que se han dedicado al estudio de la cartografía austral sudamericana. Para cuantos se han ocupado de la materia resulta evidente que este plano es una copia del mapa universal de Diego Ribero de 1529. 
lo que concierne a la presentación del estrecho de Magallanes todas se ejecutaron en cuanto a sus características distintivas ciñéndose a la imagen elaborada por García de Toreno en 1523, pero con toponimia más abundante $e^{16}$.

El mismo afamado depósito documental vaticano conserva otras dos cartas universales de la época, ca. 1529-1530, una debida a Girolamo da Verrazzano y otra de autoría desconocida, aunque se ha atribuido a Battista Agnese. Esta figura en el catálogo de registro como Bergiano Lat. II, e incluye una representación del Estrecho muy cercana a la contenida en el planisferio de Turín, con abundante toponimia, parte de la cual es poco legible.

Con estas menciones se completa la secuencia referencial acerca del gran canal interoceánico en los mapas del mundo para el lustro que siguió a las juntas de Badajoz-Elvas, cuya fuente inspirativa procuró ajustarse a la entregada originalmente en el padrón de 1523 varias veces mencionado. En la década que seguirá hasta 1540 , la revisión da cuenta de una producción cartográfica más libre en que el sector fretano de nuestro interés no ofrece una figuración mejorada y por ello más aproximada a su realidad geográfica, excepción hecha del notable trabajo del cosmógrafo Alonso de Santa Cruz con el que rematamos el período en consideración, según se verá más adelante.

1529 es el año que marca una inflexión en el curso de la historia de los intereses ultramarinos de España. En efecto, entonces se llegó a un arreglo mutuamente satisfactorio entre las dos potencias ibéricas, Portugal y España, por su contencioso de las Molucas. Ante el estancamiento de las negociaciones y los fracasos que siguieron a la expedición de Fernando de Magallanes en procura

16 Véase Martinic, 1999. Cartografía Magallánica 15231945, Ediciones de la Universidad de Magallanes, Punta Arenas, figuras 6 y 7 de la p. 6.

17 No hay certidumbre plena acerca de cuál pudo ser la primera carta universal impresa en que aparece representado el Estrecho, pues si por algún investigador se ha otorgado tal primacía al mapamundi de 1527 desaparecido de la Biblioteca Colombina al que se ha hecho mención precedente, otro especialista como es Peter Whitfield la adjudica a otra pieza contemporánea, el mapamundi ejecutado por Franciscus Monachus. Un tercero, Oswald Dreyer-Eimbcke ha afirmado que tal condición debe reconocerse al mapamundi de de la afirmación en el terreno de las reclamaciones hispanas sobre las codiciadas islas de las especias, por una parte, y la urgente necesidad de disponer de recursos financieros para atender los intereses políticos imperiales en el centro de Europa, Carlos $\mathrm{V}$ y su par el rey Juan III de Portugal arribaron a un buen acuerdo. En su virtud, España enajenó al reino luso sus reclamaciones por la suma de 300.000 escudos de oro, una cantidad enorme para su tiempo, de modo que éste se vio confirmado en su dominio sobre las islas Molucas y su riqueza natural, en tanto que aquella, su poderoso monarca en verdad pudo disponer de recursos para atender sus cada vez más complejos intereses en Europa y el Mediterráneo.

Esta circunstancia coyuntural de algún modo también modificaría la historia de la producción cartográfica al acelerar su universalización.

Un cabal ejemplo de esta tendencia se tiene en el original mapa en proyección doble cordiforme del francés Orontius Finneus titulado Nova et integra orbis descriptio. Elaborado en 1531 y editado impreso en París al año siguiente, se lo considera la primera pieza de este carácter, lo que facilitó su difusión ${ }^{17}$. No obstante su condición de mapa universal, en él el estrecho de Magallanes se advierte notorio en la sección correspondiente como punto de separación entre dos continentes, el americano de una parte, y otro descomunal de la otra presentado como Terra Australis Recenter Inventa, sed nondum plena cognita. Este estilo representativo fue continuado por el mismo autor en 1536 y más tarde, en 1538, por el holandés Gerardo Mercator quien no tardaría en adquirir fama como autor y editor de mapas.

El eco del sensacional viaje de Fernando de Magallanes y de su finalizador Elcano siguió por

Orontius Finneus recién descrito. En nuestra opinión, la primacía debiera reconocerse al denominado "Globo del Embajador", pieza elaborada hacia 1526 en Nuremberg por un cartógrafo desconocido y que incluye el trazado del viaje de circunnavegación de Magallanes, tal como más tarde lo hará Battista Agnese, de la que se conocen únicamente tres ejemplares: uno de ellos se conserva en la Universidad de Yale (Beinecke Rare Book and Manuscript Library). La denominación con la que se conoce este rarísimo mapa deriva de su reproducción hecha en el cuadro de Hans Holbein, The Ambassador (1533) que se exhibe en la National Gallery de Londres. 
entonces animando la imaginación colectiva, como lo demuestra la tan divulgada carta planisferia elaborada por el cartógrafo italiano Battista Agnese que muestra la ruta seguida por la expedición de 1519-1522, en una serie de ediciones impresas realizadas desde 1536 hasta 1543 , con reediciones posteriores. Mientras así ocurría la producción española de la década, aunque atenuada, prosiguió en la línea informativa conocida para el segundo lustro de la década precedente, en especial con los mapas de Alonso de Chaves, entre los que cabe mencionar su Carta de América y Filipinas, pieza manuscrita que presenta al Estrecho siguiendo la pauta descriptiva de Diego Ribero.

Se ha mencionado antes la internacionalización que tuvo la elaboración de mapas durante el siglo XVI gracias al potente invento de Gutenberg que facilitó la difusión de obras impresas, de grandísimo interés y demanda en círculos académicos, políticos y del empresariado económico, por la información que se brindaba acerca del progresivo mejor conocimiento de la geografía del mundo y de las perspectivas variadas que tal fenómeno sugería bajo diferentes miradas. De allí que surgieran por entonces, promediando esa centuria, nuevos centros de producción cartográfica distintos a los tradicionales españoles, portugueses, alemanes e italianos. Tales fueron los casos de los instalados en la ciudad de Dieppe, Francia, cuya actividad marcaría toda una época y especialmente en la ciudad flamenca de Amberes donde la misma alcanzaría la máxima calificación por su sorprendente desarrollo durante el curso de la segunda mitad del siglo. En ese centro de producción cartográfica y en otros de los vecinos Países Bajos, como la ciudad de Ámsterdam, la actividad elaboradora y difusora alcanzaría su período áureo durante la centuria decimoséptima. De esa manera el conocimiento sobre los territorios y mares del mundo, incluida la región meridional de América, se universalizó y estuvo cada vez más a disposición de la gente interesada.

Aunque los mapas generales abundaron por entonces, también comenzaron a elaborarse los primeros planos sectoriales de las regiones recientemente descubiertas. De ellos los primeros parecen ser los dos realizados por el maestro portugués Gaspar Viegas hacia 1537, ambos conocidos bajo idéntico nombre, Carta del Río de la Plata-Estrecho de Magallanes, atribuido por investigadores modernos. Estas piezas se conservan en repositorios de Florencia, uno en la Biblioteca Ricardiana y otro en el Archivio di State. Aunque en ellos la representación del litoral patagónico deja mucho que desear en su trazado, el concerniente al Estrecho (forma y rumbos) se ajusta al modelo definido por Ribero salvo en su parte sur en que se incluyen dos islas imaginarias hacia el oriente. La condición insular de la gran tierra del sur, entonces desconocida, se ofrece clarísima en la primera de las cartas mencionadas. La toponimia incluida nos sugiere la posibilidad de haber tenido a la vista el cartógrafo información documental de primera mano sobre el viaje de Magallanes y que por la época debía guardarse en archivos lusos.

Sin embargo de lo anterior, la primacía representativa para la región del estrecho de Magallanes debe otorgarse al mapa construido por el cosmógrafo español Alonso de Santa Cruz. Hombre versado en la geografía y en navegación, participó en la expedición de Sebastián Caboto al río de la Plata en 1530, y más tarde, en 1536, fue designado cosmógrafo mayor de la Casa de Contratación, responsabilidad que mantuvo por muchos años. Sus conocimientos y maestría en el arte cartográfico le permitieron preparar un conjunto de mapas del mundo y de Sudamérica en particular, que incluyó en un gran trabajo que llamó Islario de todas las islas del mundo. Extraviada durante siglos, esta preciada obra fue encontrada en el siglo XIX y se conserva en la Biblioteca Nacional de Madrid. Dividida en siete partes, tablas o padrones, nos interesa en especial la última, cuya fecha de elaboración se sitúa hacia 1540 y que representa a la zona austral americana entre los $48^{\circ}$ y los $54^{\circ}$ de latitud sur, aproximadamente (Fig. 3).

Trabajo notable por donde se lo mire, en él el Estrecho está bien figurado en su forma, con un litoral nororiental abundante en bahías y otro noroccidental con sucesivas profundas penetraciones tierra adentro que sugieren senos y fiordos. La costa del sur del gran canal se muestra en su parte central con dos extensos espacios marinos en los que reconocemos a los actuales bahía Inútil y fiordo del Almirantazgo; su prolongación sudoccidental muestra numerosas islas que dan cuenta de la realidad archipielágica del 
sector, condición igualmente sugerida para su parte pacífica. Incomprensible resulta, en cambio, la imaginaria continuación atlántica de la actual Tierra del Fuego, en demasía hacia el este. Exceptuando este exceso, parece evidente que tanta aproximación a la realidad geográfica -para su tiempopudo ser posible por el conocimiento que Santa Cruz pudo de tener acerca de antecedentes gráficos y escritos (planos, derroteros) procedentes de las expediciones de Magallanes y Loayza, parte de los cuales debió extraviarse hace ya mucho tiempo. En suma, la carta en consideración es una obra notable que debe ser reconocida como el primer mapa sectorial del estrecho de Magallanes, materia de relevancia indudable en la historia cartográfica chilena.

\section{LA TOPONIMIA \\ INICIAL Y SU EVOLUCIÓN}

El estrecho de Magallanes, causa $e$ inicio del interés universal por la geografía del meridión del mundo y su vasto ámbito occidental, en tanto que paso a través del mismo para conectar a los dos mayores océanos Atlántico y Pacífico, devino en su divulgación el primer accidente individualizable e individualizado. Hasta hace muy poco se aceptaba que en un principio se lo conociera como Estrecho de las Once Mil Vírgenes, según Antonio Pigafetta, denominación tomada de la del cabo homónimo que señala su acceso por el noroeste y ésta, a su vez, por la festividad del día de su hallazgo, 21 de octubre, de acuerdo con el santoral cristiano (conmemoración del martirio de Santa Úrsula y de las Once Mil Vírgenes). Pero el mismo cronista da cuenta en su relación del nombre Canal de Todos los Santos dado por Fernando de Magallanes al Estrecho mientras promediaba su navegación exploratoria y después, al poner término a esa parte de su escrito, agrega que... llamamos a ese estrecho el Estrecho Patagónico, topónimo reafirmado en su mapa ya comentado. De esa triple denominación inicial pareció predominar la

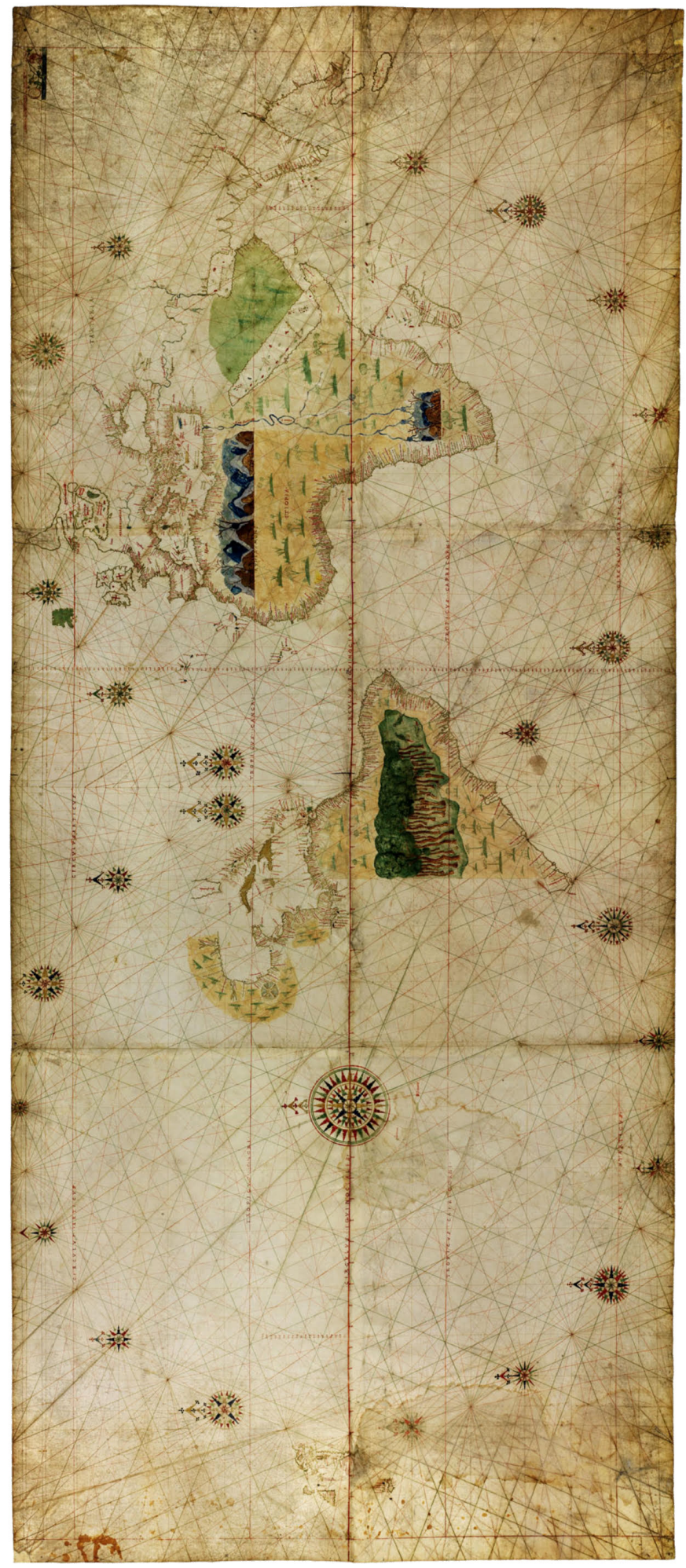

Fig. 3. Alonso Santa Cruz, Islario general de todas las islas del Mundo. Biblioteca Nacional de España. 
asignada por Magallanes, como que fue recogida en los planisferios de Turín, de Ribero (1525, 1527 y 1529), de Toreno, Caboto y Finneus entre otros.

Pero a poco andar, en 1527, el mapamundi atribuido a Diego Ribero compartiría aquella denominación con la de Estrecho de Fernam de Magallanes y que ya como topónimo único no demoraría en su aceptación y uso hasta nuestros días, como merecida denominación para memoria del gran navegante que lo revelara para la geografía del mundo. Sin embargo, la primacía en esta denominación debe reconocerse al cartógrafo Lorenz Fries, de Estrasburgo, autor de una carta manuscrita datada hacia 1525 y recientemente dada a conocer al mundo académico ${ }^{18}$. A este mismo autor debe acreditársele el efímero topónimo Tierra de Vespucio, para una parte del territorio austral del Estrecho, y que valoramos como expresión del reconocimiento que pudo tener Magallanes hacia el ilustre florentino por su carácter de precursor en la ruta que resultó determinante para su memorable hallazgo histórico.

Aparte de la triple toponimia inicial, otros nombres fueron asignados por entonces al Estrecho, todos efímeros por peregrinos: Estrecho de Sant Anton (Juan Vespucio 1526), Estrecho de la Victoria, Estrecho de los Malucos, y más tardíamente, Estrecho del Perú, por otros autores.

Tocante a los accidentes correspondientes a los litorales e interior del Estrecho, Pigafetta únicamente menciona los de los cabos Once Mil Vírgenes y Deseado, éste para la individualización de la extremidad sudoccidental del gran canal. Estos topónimos fueron recogidos por el mapamundi de Turín de 1523. La expedición de Loayza que siguió a la travesía del descubrimiento no dejó mapa alguno conocido en el Estrecho, pero sí una relación de su paso por él en 1526, obra del piloto Hernando de la Torre. En ella se consignan las bahías de las Virgenes, que debe suponerse inmediata al cabo homónimo y hacia el interior del Estrecho; de la Victoria, Puerto de la Concepción y Puerto de las Sardinas, topónimos que sugieren su origen en la travesía magallánica y que pudieron ser mencionados en alguna relación de la misma perdida desde hace siglos, y que deben ser identificados con las actuales bahía Posesión, rada Pelícano y bahía Fortescue, respectivamente. Se añaden otros nombres para accidentes al interior en la costa norte, como Cabo del Descanso, Angla de San Jorge y Buen Puerto, todos para el sector occidental y de vigencia efímera, amén del cabo Deseado.

La costa sur del Estrecho se visibiliza toponímicamente con los mapas de Ribero (Castiglioni, 1525) y Toreno (Salviati, 1525), que consignaron las denominaciones tierra de los humos, lago de los estrechos, tierra de los fuegos, sierras nevadas, campana de Roldán e Yslas nevadas, en su mayoría de carácter general más que específico, excepción hecha de la segunda claramente identificable con la actual bahía Inútil, y la penúltima con la que se nombró la eminencia litoral desde la que, de acuerdo con la tradición, el flamenco Roldán de Argote avizorara el término del Estrecho. Todos estos nombres fueron recogidos de modo casi invariable por la cartografía de los tres lustros siguientes hasta 1540. Las excepciones se dieron con los topónimos c. (cabo) del norte y b. del norte, que entendemos referidos a un mismo accidente de la costa septentrional en sitio hoy indeterminable. Ambos fueron recogidos bajo la última forma en todos los mapas de Ribero y otros de la tercera década.

Las novedades registradas en la siguiente corrieron por cuenta de Gaspar Viegas y Alonso de Santa Cruz. Al cartógrafo luso se debe la recuperación del topónimo histórico del puerto de las Sardinas y el añadido del curioso testa de balena (cabeza de ballena), que por su ubicación y por la forma natural que sugiere su perfil identificamos como la primera denominación para el cabo-promontorio después llamado Froward, conspicuo morro litoral terminal del continente americano; hay además otro topónimo que nos ha resultado ilegible.

Santa Cruz, por fin, consiguió para su tiempo y la posteridad quince topónimos, a saber: estrecho de Magallanes, c. de las Once Mil Virgenes, b. de la Victoria, b. del Isleo (posiblemente la actual rada Pelícano), $b$. del Norte (¿bahía San Juan de la Posesión?); canal de Todos los Santos, y archipiélago del cabo Deseado, topónimo este identificatorio del islario adyacente, para el sector septentrional; y Tierra de los Humos, lago de los Estrechos,

18 Véase la nota $\mathrm{N}^{\circ} 1$. 
Tierra de los Fuegos, b. Grande (seno Almirantazgo), sierras nevadas, campana de Roldán, ys. Nevadas y c. Deseado, para la parte meridional. Con el añadido de islas de los Pájaros para el conjunto de las actuales Magdalena y Marta en el comienzo del paso Ancho, para el que no hemos encontrado sustento cartográfico, pero su referencia escrita, se completa la toponimia inicial $\mathrm{u}$ original del estrecho de Magallanes, período que cerramos para su debida comprensión en 1540 con la carta famosa de Santa Cruz.

\section{CONCLUSIÓN}

En la geografía, como suele darse en todo proceso, el correspondiente tiempo fundacional está determinado por las informaciones que lo caracterizan para la historia de modo general pero preciso. Así ha ocurrido en lo que se refiere al estrecho de Magallanes -imagen cartográfica y toponimia original- durante el lapso comprendido entre la primera representación cartográfica aceptada como tal, 1523, y 1540, año en que el maestro Alonso de Santa Cruz fechó el que se conoce como el primer mapa sectorial del gran canal interoceánico chileno.

El mérito de tal condición puede apreciarse además por el hecho de su perdurabilidad: su doble información no fue mejorada en el transcurso del medio siglo que siguió al trabajo de Santa Cruz. En efecto, tanto lo fue que recién en los comienzos del siglo XVII y gracias a los determinantes reconocimientos y aportes hechos por las expediciones holandesas de 1599-1600 (Cordes y van Noort), recogidos y difundidos por eminentes cartógrafos profesionales del mismo origen nacional, el gran canal descubierto por Magallanes en 1520 pudo ser conocido documentalmente con una sorprendente cercanía en rumbos y forma a los de su realidad geográfica. Esta tarea, relevante por donde se la mire, se complementaría durante el mismo siglo con posteriores contribuciones holandesas, francesas $e$ inglesas principalmente, éstas realizadas entre los siglos XVIII y XX.

Tocante a la toponimia, la innovación correría por cuenta de navegantes españoles, Juan Ladrillero (1557-1558) y especialmente Pedro Sarmiento de Gamboa (1579-1584), cuyas contribuciones, importantes sin duda, tardaron sin embargo en ser conocidas por causa de la política secretista de la corona española que quiso reservar esas informaciones para su uso exclusivo. Una información tan valiosa demoraría hasta dos siglos en ser conocida después de ser originada y de la misma solo hubo referencias puntuales que de algún modo pudieron escapar del control oficial ${ }^{19}$.

\section{AGRADECIMIENTOS}

Agradezco al profesor Rodrigo Moreno Jeria por haber compartido conmigo los mapas que se han publicado en este artículo. Asimismo, José María García Redondo, por el mapa de Alonso de Santa Cruz.

\section{BIBLIOGRAFÍA}

Barber, P. (Ed.) (2005). The Map Book. New York: Levenger Press.

Barreiro-Meiro, R. (1975). El Pacífico y el estrecho de Magallanes en la cartografía del siglo XVI. En A viagem de Fernao de Magalhaes e a questao das Molucas. Actas do II Coloquio Luso-Espanhol de Historia Ultramarina. Lisboa.

Cerezo Martínez, R. (1994). La cartografía náutica española en los siglos XIV, XV y XVI. Madrid: Consejo Superior de Investigaciones Científicas.

Martín Merás, L. (1993). Cartografía Marítima Hispana. La imagen de América. Barcelona: Lunwerg Editores.

Martinic, M. (1999). Cartografía Magallánica 1523-1945. Punta Arenas: Ediciones de la Universidad de Magallanes.
19 Confirma nuestra conjetura la observación personal que hicimos en abril de 2018 del Atlas Náutico de 1562 atribuido a Bartolomé de Oliva, valiosa pieza cartográfica manuscrita obsequiada a la Pontificia Universidad Católica de Chile por el Papa Francisco durante su visita al país en enero del mismo año. Pues bien, uno de los nueve folios que lo componen representa a América del Sur, incluido el estrecho de Magallanes, mapa que en su margen inferior derecho incluye una anotación que da cuenta de haberse considerado para el trazado de la costa sudoccidental las noticias aportadas por la reciente navegación exploratoria comandada por Juan Ladrillero. Este atlas (del que al parecer se hicieron varias copias) está registrado en la Biblioteca Apostólica Vaticana como Urb. Lat. 283. 
Martinic, M. (2005). El protomapa de Chile. Boletín de la Academia Chilena de la Historia, 114, 175-182.

Martinic, M. (2016). Una travesía memorable. Hallazgo y navegación del estrecho de Magallanes. Punta Arenas: Aguas Magallanes.

Martinic, M. (2017). Tierra de Vespucio. Consideraciones sobre una curiosa representación del estrecho de Magallanes en la Tabula Moderna Alterius Hemisphaerii, un mapa recién conocido del siglo XVI. Magallania 45(1), pp. 5-14.
Muller, F. (2012). Tabula Moderna Alterius Hemisphaerii. The oldest surviving map of the Pacific? The Globe. Journal of the Australian and New Zeland Map Society Inc., 71.

Pigafetta, A. (1985). Primer viaje alrededor del mundo. Edición de Leoncio Cabrero. Madrid: Historia 16.

Zweig, S. (1972). Magallanes. Historia del primer viaje alrededor del mundo. Barcelona: Editorial Juventud S.A. 